\title{
Valores energéticos e composição bromatológica dos fenos de jureminha, feijão bravo e maniçoba para aves ${ }^{1}$
}

\author{
Fernando Guilherme Perazzo Costa ${ }^{2}$, Cleber Franklin Santos de Oliveira ${ }^{3}$, Leilane Rocha \\ Barros $^{4}$, Edson Lindolfo da Silva ${ }^{5}$, Raul da Cunha Lima Neto ${ }^{6}$, José Humberto Vilar da Silva ${ }^{7}$ \\ 1 Trabalho de Iniciação Científica do segundo autor, PIBIC/UFPB/CNPq \\ 2 DZ/CCA/UFPB, Areia-PB. \\ ${ }^{3}$ Graduando em Zootecnia, DZ/CCA/UFPB. \\ 4 Pós-Graduação em Zootecnia, FCAV/UNESP \\ ${ }^{5}$ Pós-Graduação em Zootecnia, UFLA/MG. \\ ${ }^{6}$ Pós-Graduação em Zootecnia, CCA/UFPB. \\ 7 DAP/CFT/UFPB, Bananeiras-PB.
}

RESUMO - Um experimento foi conduzido para determinação dos valores de energia metabolizável aparente (EMA) e energia metabolizável aparente corrigida pelo balanço de nitrogênio (EMAn) e da composição química dos fenos de feijãobravo (FFB), jureminha (FJ) e maniçoba (FM) para uso na alimentação de aves. Duzentos e oitenta pintos com 17 dias de idade foram distribuídos aleatoriamente em sete tratamentos, em esquema fatorial $3 \times 2+1$ (três tipos de feno x dois níveis de substituição da dieta-referência + dieta-referência), com quatro repetições de dez aves. Os níveis de EMA e EMAn dos fenos de jureminha, feijão-bravo e maniçoba foram 3.205 e 2.911, 2.990 e 2.648, 2.728 e 2.419, determinados com 15\% de substituição da dieta-referência pelo alimento e 2.678 e 2.371, 2.875 e 2.523, 2.277 e 1.956 com substituição de 30\%, respectivamente, e evidenciaram influência do nível de substituição da dieta-referência sobre os valores de EMA e EMAn das dietas. Os valores de EMA e EMAn, para aves, dos fenos de jureminha e feijão-bravo são superiores aos do feno de maniçoba.

Palavras-chave: alimento alternativo, forrageiras nativas, frangos de corte

\section{Energy values and chemical composition of jureminha, bravo beans and maniçoba hays for poultry}

\begin{abstract}
An experiment was carried out to determine the apparent metabolizable energy (AME), apparent metabolizable energy corrected for nitrogen balance (AMEn) and chemical composition of bravo bean hay (HBB), jureminha hay (HJ) and maniçoba hay (HM) for use in birds feeding. Two hundred and eighty, 17 days old broiler chicks were distributed according to a completely randomized design, into seven treatments organized in a factorial arrangement $3 \times 2+1$ (three hay types $\mathrm{x}$ two levels of substitution of the reference diet + reference diet), with four repetitions of 10 birds each. The contents of AME and AMEn of jureminha, bravo bean and maniçoba hays were 3.205 and 2.911, 2.990 and 2.648, 2.728 and 2.419, respectively, when the ingredients test substituted $15 \%$ of the reference diet, and 2.678 and $2.371,2.875$ and $2.523,2.277$ and 1.956, respectively, when the ingredients test substituted $30 \%$ of the reference diet. Therefore, the level of substitution of the reference diet by the ingredient test affects AME and AMEn. Jureminha and bravo bean hays presented higher AME and AMEn values than maniçoba hay for bird feeding.
\end{abstract}

Key Words: alternative food, broiler chicks, native forage

\section{Introdução}

A criação alternativa de frangos no Brasil vem crescendo desde a década de 90 . O uso de ingredientes alternativos nas dietas tem sido bastante estudado com a finalidade de diminuir o custo das dietas. O número de produtores envolvidos nessa atividade está se multiplicando, como resultado da implementação de iniciativas à criação baseadas em paradigmas ecológicos e oferta de produtos mais saudáveis. Atualmente, observa-se grande intensificação no desenvolvimento de métodos mais naturais de criação das aves em quase todo o mundo. Além disso, a criação alternativa no Brasil parece ser uma atividade interessante para pequenos e médios produtores que podem atender a um nicho de mercado exigente, tornando essa atividade cada vez mais tecnificada, eficiente e rentável.

Silva \& Nakano (1998) afirmaram haver diferenças no sistema caipira atribuídas à ingestão, pelas aves, de 
determinados alimentos, inclusive pastagem, verduras, insetos e minhocas, encontrados em grande quantidade no sistema semi-intensivo de criação.

Fatores como a exigência das aves e a composição química do alimento devem ser considerados para que a introdução de alimentos alternativos em dietas seja feita em quantidades suficientes para suprir as exigências nutricionais dos animais.

Na região do semi-árido nordestino, é conhecida a riqueza vegetativa de algumas leguminosas, entretanto, seu aproveitamento destina-se, em sua maioria, à alimentação dos animais ruminantes. Na alimentação de aves, o grande inconveniente dessas forrageiras seria a quantidade de fibras e a presença de fatores antinutricionais, como o tanino, composto fenólico de alto peso molecular que contém grupos hidroxifenóis.

Segundo Chubb (1982), os fatores antinutricionais são de três tipos: substâncias que prejudicam a digestibilidade ou a utilização metabólica das proteínas (inibidores de enzimas digestivas, lecitinas ou hemaglutininas, saponinas e compostos fenólicos); substâncias que reduzem a solubilidade ou interferem na utilização dos minerais (ácido fítico, ácido oxálico, glicosinolatos e gossipol); e substâncias que inativam ou aumentam as necessidades de algumas vitaminas (antivitaminas A, D, E, K, antivitaminas tiamina, ácido nicotínico, piridoxina e cianocobalamina). Na literatura, são escassas ou inexistentes pesquisas identificando a composição química dessas leguminosas, sua digestibilidade e seu potencial de utilização como ingrediente alternativo de dietas para aves.

De acordo com Bokanga (1994), existem 2.000 espécies de plantas cianogênicas conhecidas, mas em nenhuma delas o ácido cianídrico (HCN) é produzido diretamente ou armazenado na planta em qualquer estádio de desenvolvimento. A maniçoba apresenta, na planta verde em início de brotação, teor médio de HCN de $1.000 \mathrm{mg} / \mathrm{kg}$ de MS. Portanto, se o animal consumir grande quantidade, pode sofrer intoxicação em poucos instantes. Por outro lado, quando triturada e seca (fenada), o teor de HCN reduz para menos de $300 \mathrm{mg} / \mathrm{kg}$ de MS, quantidade insuficiente para provocar qualquer sintoma de intoxicação em animais, mesmo que consumida em grande quantidade e por muito tempo (Araújo \& Cavalcanti, 2002).

Para boa utilização das forrageiras na dieta, busca-se constantemente formular dietas eficientes e de baixo custo, o que aumenta a necessidade de pesquisas para determinação da composição química e da digestibilidade dos nutrientes dos alimentos utilizados nas formulações, visando obter maior eficiência no desempenho animal (Nunes et al., 2005).
O principal aspecto para a formulação de dietas é a determinação da energia metabolizável, pois, a partir dos valores energéticos, é estimado o nível de inclusão do alimento às dietas. A formulação correta de dietas balanceadas é fundamental, pois possibilita o suprimento das exigências nutricionais dos animais permitindo que expresse ao máximo seu potencial genético.

Segundo Emmans (1995), para manter o balanço nutricional estável a ave precisa de nutrientes que devem ser fornecidos em níveis adequados pela dieta, visando seu melhor aproveitamento para ganho de peso ou produção de ovos. Albino et al. (1992) observaram que, para se obter sucesso na formulação de rações para aves, um dos aspectos mais importantes é o conhecimento preciso do conteúdo energético dos alimentos, o que possibilita o fornecimento adequado de energia para as aves em cada fase de seu ciclo de vida.

A energia metabolizável é a melhor forma de expressar a energia disponível para as aves. Assim, é imprescindível a determinação dos valores de energia metabolizável aparente (EMA) e energia metabolizável aparente corrigida (EMAn) dos ingredientes, bem como a composição físicoquímica dos alimentos. Brum et al. (2000) enfatizaram a importância da contínua avaliação dos ingredientes para manter atualizado um banco de dados, possibilitando melhorar as estimativas dos valores de energia metabolizável de alimentos utilizados em dietas para aves.

Nesta pesquisa, determinaram-se os valores de energia metabolizável aparente (EMA) e energia metabolizável aparente corrigida pelo balanço de nitrogênio (EMAn) e a composição química dos fenos de maniçoba, jureminha e feijão-bravo para alimentação de aves.

\section{Material e Métodos}

O experimento foi conduzido no Setor de Avicultura do Departamento de Zootecnia do Centro de Ciências Agrárias da Universidade Federal da Paraíba (DZ/CCA/UFPB), situado na cidade de Areia na microrregião do Brejo Paraibano.

Foram utilizados fenos de três plantas forrageiras: maniçoba (Manihot pseudoglaziovii Pax \& Hoffman), jureminha (Desmanthus virgatus) e feijão-bravo (Capparis flexuosa). As plantas foram coletadas na microrregião do Cariri Paraibano e no Curimatau Paraibano, evitando-se colher as partes lignificadas.

Foram utilizados 280 pintos de corte da linhagem Ross, com 17 dias de idade e peso médio de $470 \mathrm{~g}$. Os pintos foram criados desde o $1 \underline{0}$ dia de idade em uma bateria de gaiolas de arame galvanizado. Cada gaiola tinha bebedouro tipo calha e um comedouro individual na parte frontal. Até o 170 
dia de idade, as aves foram alimentadas com dieta inicial para frangos de corte.

O período experimental teve duração de dez dias - cinco para adaptação e cinco para coleta. O ensaio biológico foi constituído de uma dieta-referência (Tabela 1) e seis dietasteste, constituídas pela substituição da dieta-referência por três tipos de feno (jureminha, feijão-bravo e maniçoba), em dois níveis de substituição (15 e 30\%). A composição da dietareferência foi calculada segundo recomendações de Rostagno et al. (2000). O delineamento adotado foi o inteiramente casualizado, com sete tratamentos, em esquema fatorial $3 \times 2+1$, com quatro repetições e dez aves por unidade experimental.

Tabela 1 - Composição percentual da dieta-referência Table 1 - Percentage composition of the reference diet

\begin{tabular}{|c|c|}
\hline $\begin{array}{l}\text { Ingrediente } \\
\text { Ingredient }\end{array}$ & $\%$ \\
\hline Milho (Corn) & 61,014 \\
\hline Farelo de soja (Soybean meal) & 34,114 \\
\hline Calcário (Limestone) & 1,021 \\
\hline Fosfato bicálcico (Dicalcium phosphate) & 1,763 \\
\hline DL-metionina (DL-methionine) 99\% & 0,196 \\
\hline L-lisina-HCl (L-lysine) 78,4\% & 0,159 \\
\hline Cloreto de colina (Coline chloride) $70 \%$ & 0,050 \\
\hline Óleo vegetal (Vegetable oil) & 0,873 \\
\hline Sal (Salt) & 0,502 \\
\hline Mistura vitamínica ${ }^{2}$ (Vitamin mix) & 0,050 \\
\hline Mistura mineral $^{3}$ (Mineral mix) & 0,100 \\
\hline Anticoccidiano 4 (Anticoccidial) & 0,010 \\
\hline Promotor de crescimento ${ }^{5}$ (Growth promoter) & 0,050 \\
\hline Promotor de crescimento ${ }^{6}$ (Growth promoter) & 0,002 \\
\hline Antioxidante $^{7}$ (Antioxidant) & 0,010 \\
\hline Inerte (Inert) & 0,086 \\
\hline Total & 100 \\
\hline
\end{tabular}

Composição calculada

Calculated composition

PB (CP) (\%)

EM (ME) $(\mathrm{kcal} / \mathrm{kg})$

MS (DM) (\%)

FB $(C F)(\%)$

Metionina digestível (Digestible methionine) (\%)

21,400

3.000

86,02

3,60

0,497

Metionina+cistina digestível (Digestible Met+Cys) (\%) 0,813

Lisina digestível (Digestible lysine) (\%) 1,154

Treonina digestível (Digestible threonine) (\%) $\quad 0,733$

Triptofano digestível (Digestible tryptophan) (\%) 0,236

Ca $(\%)$

P disponível (Available P) (\%)

0,960

0,450

0,222

0,347

0,923

$\mathrm{Cl}(\%)$

1,841

Ácido linoléico (Linoleic acid) (\%)

${ }^{1}$ Valores calculados segundo Rostagno et al. (2000) (Calculated values according with Rostagno et al., 2000).

2 Composição por kg produto, suplemento de vitaminas (Vitamin supplement, composition per $\mathrm{kg}$ of product): vit. A - $10.000 \mathrm{UI}$; vit. $\mathrm{D}_{3}-2.000 \mathrm{UI}$; vit. E -

$30 \mathrm{UI}$; vit. $\mathrm{B}_{1}-2 \mathrm{mg}$; vit. $\mathrm{B}_{6}-3 \mathrm{mg}$; ác. pantotênico (pantothenic acid) -

$12 \mathrm{mg}$; biotina (biotin) - 0,1 g; vit. $\mathrm{K}_{3}-3 \mathrm{mg}$; ac. fólico (folic acid) - $1 \mathrm{mg}$; ac. nicotínico (nicotinic acid) - $50 \mathrm{mg}$; vit. $\mathrm{B}_{12}-0,015 \mathrm{mg}$.

3 Composição básica por $\mathrm{kg}$ do produto, suplemento de minerais (Mineral supplement, composition per $\mathrm{kg}$ of product): Se - 0,25 mg; Mn - $106 \mathrm{mg}$; Fe -

$100 \mathrm{mg}$; Cu - $20 \mathrm{mg}$; Co - $2 \mathrm{mg}$; I $-2 \mathrm{mg}$; excipiente q.s.p. $-1.000 \mathrm{~g}$.

${ }^{4}$ Coban, ${ }^{5}$ Enradin, 6 Colistin, ${ }^{7}$ Etoxiquin.
Após o período de adaptação, as dietas foram adicionadas de $2 \%$ de óxido férrico como marcador no primeiro e no último dia de coleta total de excretas. As coletas foram realizadas duas vezes ao dia, às 8 e $17 \mathrm{~h}$. Para evitar perdas, utilizaram-se bandejas cobertas com plástico, colocadas sob cada compartimento das gaiolas. Ao término do período experimental, determinou-se a quantidade de dieta consumida por unidade experimental durante os cinco dias de coleta.

As excretas foram acondicionadas em sacos plásticos devidamente identificados e armazenadas em freezer até o final do período experimental. Posteriormente, foram descongeladas, homogeneizadas e pesadas. Amostras foram retiradas e analisadas após pré-secagem em estufa ventilada a $55^{\circ} \mathrm{C}$, para cálculo dos teores de MS, EB, FB e PB, conforme descrito por Silva \& Queiroz (2002).

Os valores de EB da dieta-referência, das dietas-teste e das excretas foram determinados por meio de uma bomba calorimétrica adiabática (Parr Instruments Co). Os valores de energia metabolizável aparente (EMA) e energia metabolizável aparente corrigida pelo balanço de nitrogênio (EMAn) dos fenos foram estimados utilizando-se a equação de Matterson et al. (1965).

As análises estatísticas foram realizadas pelo programa estatístico SAEG (Sistema para Análise Estatística e Genética), desenvolvido na Universidade Federal de Viçosa (1999).

\section{Resultados e Discussão}

A composição química das dietas avaliadas é apresentada na Tabela 2. Os valores de MS e PB do feno de jureminha foram próximos e o de cinzas, um pouco superior (7,17\%) aos encontrados por Figueiredo et al. (2000), de 84,07 e $12,42 \%$, respectivamente, no feno processado desta forrageira - no experimento realizado por esses autores, o corte das plantas foi feito aos 395 dias de crescimento.

No feno de feijão-bravo, os valores de FB (37,10\%), PB (16,28\%) e cinzas (92,92\%) foram superiores aos determinados por Araújo et al (2000), de 31,92; 12,5 e 90,22\%. O teor de MS (92,8\%), no entanto, foi inferior ao determinado por esses autores $(86,77 \%)$.

Estudando a composição química do feno de maniçoba, Barros et al. (1990) encontraram 93,30\% de MS e 12,00\% de PB, enquanto Salviano \& Nunes (1991) registraram valores de 13,52; 16,52 e 8,40\% para PB, FB e cinzas, respectivamente. Araújo et al. (1996) obtiveram valores de 89,99\% de MS, 9,26\% de PB e 28,13\% de FB. Essas diferenças na composição química dos fenos podem estar relacionadas ao local de colheita da planta e à idade de corte e às partes da planta utilizadas para confecção do feno. 
Tabela 2 - Composição química dos fenos

Table 2 - Chemical composition of the forage hays

\begin{tabular}{lcccccc}
\hline Feno & MS (\%) & FB (\%) & N (\%) & PB (\%) & \multicolumn{2}{c}{ Cinzas (\%) } \\
Hay & DM & CF & & CP (kcal/kg) & Ash \\
\hline Jureminha (Desmanthus virgatus) & 84,77 & 37,29 & 1,78 & 11,13 & 4,57 & 4.390 \\
Feijão-bravo (Capparis flexuosa) & 86,73 & 37,10 & 2,61 & 16,28 & 7,08 & 4.542 \\
Maniçoba (Manihot pseudoglaziovii) & 86,29 & 17,83 & 2,88 & 18,03 & 9,73 & 4.390 \\
\hline
\end{tabular}

Os valores médios de energia metabolizável aparente (EMA) e aparente corrigida pelo balanço de nitrogênio (EMAn) dos alimentos, com base na matéria natural e na MS, determinados nas dietas com 15 e 30\% de substituição da dieta-referência, são descritos na Tabela 3.

No nível de $15 \%$ de substituição, os valores de EMA e EMAn dos fenos de jureminha, feijão-bravo e maniçoba foram 3.205 e 2.911, 2.990 e 2.648, 2,728 e 2.419, enquanto, no nível de 30\% de substituição, foram 2.678 e $2.371,2.875$ e 2.523 , 2.277 e 1.956 , respectivamente.

Os níveis de substituição da dieta-referência pelos alimentos alternativos tiveram efeito significativo $(\mathrm{P}<0,05)$ sobre os valores de EMA e EMAn na matéria natural e na MS, bem como no coeficiente de digestibilidade da EB (CDEB). Os fenos de jureminha e feijão-bravo apresentaram valores de EMA e EMAn e CDEB superiores aos do feno de maniçoba $(\mathrm{P}<0,05)$.

Tabela 3 - Valores de energia metabolizável aparente (EMA) e energia metabolizável aparente corrigida (EMAn) dos fenos Table 3 - Values of apparent metabolizable energy (AME) and corrected apparent metabolizable energy (AMEn) of hays

\begin{tabular}{|c|c|c|c|}
\hline \multirow[t]{2}{*}{$\begin{array}{l}\text { Feno } \\
\text { Hay }\end{array}$} & \multicolumn{2}{|c|}{$\begin{array}{c}\text { Nível de substituição (\%) } \\
\text { Replacement level }\end{array}$} & \multirow[t]{2}{*}{$\begin{array}{l}\text { Média } \\
\text { Mean }\end{array}$} \\
\hline & 15 & 30 & \\
\hline
\end{tabular}

Jureminha (Desmanthus virgatus) Feijão-bravo (Capparis flexuosa)

Maniçoba (Manihot pseudoglaziovii) Média (Mean)

$\mathrm{CV}$

Jureminha (Desmanthus virgatus) Feijão-bravo (Capparis flexuosa) Maniçoba (Manihot pseudoglaziovii) Média (Mean)

CV

Jureminha (Desmanthus virgatus) Feijão-bravo (Capparis flexuosa) Maniçoba (Manihot pseudoglaziovii) Média (Mean)

$\mathrm{CV}$

Jureminha (Desmanthus virgatus) Feijão-bravo (Capparis flexuosa) Maniçoba (Manihot pseudoglaziovii) Média (Mean)

$\mathrm{CV}$

\section{Jureminha (Desmanthus virgatus)}

Feijão-bravo (Capparis flexuosa)

Maniçoba (Manihot pseudoglaziovii)

Média (Mean)

CV
EMA (kcal/kg de MN)

$2.990 \pm 144$
$3.205 \pm 22$

$2.728 \pm 153$

$2.97 \mathrm{a}$

5,26
$2.941 \mathrm{~A}$

$2.932 \mathrm{~A}$

$2.502 \mathrm{~B}$

$875 \pm 230$

$2.277 \pm 32$

2609b

EMAn (kcal/kg de MN)

$2.911 \pm 23$

$2.648 \pm 173$

$2.419 \pm 153$

2659 a

5,94

EMA (kcal/kg de MS)

$3.781 \pm 24$

$3.447 \pm 153$

$3.161 \pm 165$

$3.463 \mathrm{a}$

5,24

EMAn (kcal/kg de MS)

$3434+24$

$3053 \pm 153$

$2803 \pm 165$

$3.096 \mathrm{a}$

5,92
$2.371 \pm 174$

$2.523 \pm 230$

$1.956 \pm 34$

$2283 b$
$2.640 \mathrm{~A}$

$2.585 \mathrm{~A}$

2.187B
$3.470 \mathrm{~A}$

$3.381 \mathrm{~A}$

$2.900 \mathrm{~B}$
Médias seguidas de letras diferentes diferem $(P<0,05)$ pelo teste Tukey.

Means followed by different letters differ $(P<0.05)$ by Tukey test. 
A inclusão dos fenos de forrageiras nativas nas dietasteste, principalmente os fenos de jureminha e feijão-bravo, refletiu no consumo de alimento pelos animais, que diminuiu à medida que aumentou o nível de inclusão desses fenos nas dietas. Segundo Farrel et al. (1991), os valores de EMAn e EMA podem ser influenciados pelo consumo de alimento, que pode interferir nas perdas energéticas metabólicas e endógenas.

Os resultados obtidos neste estudo corroboram a afirmativa de Sibbald \& Wolynetz (1985), que relataram que os valores de EMAn inferiores aos de EMA são reflexo do balanço positivo de nitrogênio atribuído à idade das aves.

Os diferentes valores de EMA dos ingredientes entre os tratamentos também podem estar relacionados às diferenças na composição química, na granulometria e no nível de inclusão do alimento (Penz Jr. et al., 1999), que refletem no tempo de passagem do alimento no trato gastrointestinal, determinando melhor aproveitamento da energia proveniente da fibra na dieta, o que pode estar relacionado ao maior tamanho relativo do ceco, resultando em maior digestibilidade da fibra (Murakami \& Ariki, 1998).

\section{Conclusões}

Os níveis de energia metabolizável aparente (EMA) e energia metabolizável aparente corrigida (EMAn) em kcal/kg de matéria natural dos fenos de jureminha, feijão-bravo e maniçoba foram 3.205 e 2.911, 2.990 e 2.648, 2.728 e 2.419, no nível de $15 \%$ de substituição e de 2.678 e 2.371, 2.875 e 2.523, 2.277 e 1.956 , com substituição de 30\% da dieta-referência. Esses valores evidenciam a influência do nível de substituição da dieta-referência pelos alimentos alternativos sobre os valores de EMA e EMAn da dieta. Os fenos de jureminha e feijão-bravo apresentaram valores de EMA e EMAn superiores aos do feno de maniçoba.

\section{Agradecimento}

Banco do Nordeste do Brasil/FUNDECI/ETENE.

\section{Literatura Citada}

ALBINO, L.F.T.; ROSTAGNO, H.S.; TAFURI, M.L. et al. Determinação dos valores de energia metabolizável aparente e verdadeira de alguns alimentos para aves usando diferentes métodos. Revista Brasileira de Zootecnia, v.21, p.10471058, 1992

ARAÚJO, E.C.; VIEIRA, M.E.Q.; CARDOSO, G.A.C. Valor nutritivo e consumo voluntário de forrageiras nativas da região semiárida do estado de Pernambuco. VI - Feijão-bravo (Capparis flexuosa, L.). In: REUNIÃO ANUAL DA SOCIEDADE BRASILEIRA DE ZOOTECNIA, 33., 1996, Fortaleza. Anais... Fortaleza: Sociedade Brasileira de Zootecnia, 1996. p.257-259.
ARAÚJO, G.G.L.; CAVALCANTI, J. Potencial de utilização da maniçoba. SIMPÓSIO PARAIBANO DE ZOOTECNIA, 2., 2002, Areia. Anais... Areia: Simpósio Paraibano de Zootecnia/ Gmosis, 2002. (CD-ROM).

BARROS, N.N.; SALVIANO, L.M.C.; KAWAS, J.R. Valor nutritivo da maniçoba para caprinos e ovinos. Pesquisa Agropecuária Brasileira, v.25, n.3, p.387-392, 1990.

BOKANGA, M. Processing of cassava leaves for human consumption. Acta Horticulturae, v.375, p.203-207, 1994.

BRUM, P.A.R.; ZANOTTO, D.L.; LIMA, G.J.M.M. et al. Composição química e energia metabolizável de ingredientes para aves. Pesquisa Agropecuária Brasileira, v.35, n.5, p.995-1002, 2000.

CHUBB, L.G. Anti-nutritive factors in animal feedstuffs. In: HARESING, W. (Eds.) Studies in the agricultural and food sciences butterworths. Recent advances in animal nutrition. Philadelphia: W. Haresign Butterworths, 1982. p.21-37.

EMMANS. G.C. Problems in modeling the growth of poultry. World Poultry Science Journal, v.51, p.77-89, 1995.

FARREL, D.J.; THOMPSON, E.; PREEZ, J.J. The estimation of endogenous excreta and the measurement of metabolizable energy in poultry feedstuffs using four feeding systems, four assay methods and four diets. British Poultry Science, v.32, n.3, p.483-499, 1991

FIGUEIREDO, M.V.; GUIM, A.; PIMENTA FILHO, E.C. et al. Avaliação da composição bromatológica e digestibilidade "in vitro" do feno de Desmanthus virgatus. In: REUNIÃO ANUAL DA SOCIEDADE BRASILEIRA DE ZOOTECNIA, 37., 2000, Viçosa, MG. Anais... Viçosa, MG: Sociedade Brasileira de Zootecnia, 2000. p.29.

MATTERSON, L.D.; POTTER, L.M.; STUTZ, M.W. et al. The metabolizable energy of feed ingredientes for chickens. Connecticut: Agricultural Experiment Station, 1965. p. 3-11. (Research Report, 7).

MURAKAMI, A.E.; ARIKI, J. Produção de codornas japonesas. Jaboticabal: Fundação de Apoio à Pesquisa, Ensino e Extensão, 1998. 79p.

NUNES, R.V.; POZZA, P.C.; NUNES, C.G.V. et al. Valores energéticos de subprodutos de origem animal para aves. Revista Brasileira de Zootecnia, v.34, n.4, p.1217-1224, 2005.

PENZ JR., A.M.; KESSLER, A.M.; BRUGALLI, I. Novos conceitos de energia para aves. In: SIMPÓSIO INTERNACIONAL SOBRE NUTRIÇÃO DE AVES, 1999, Campinas. Anais... Campinas: Fundação Apinco de Ciência e Tecnologia Avícola, 1999 p.1-24.

ROSTAGNO, H.S.; ALBINO, L.F.T.; DONZELE, J.L. et al. Tabelas brasileiras para aves e suínos: composição de alimentos e exigências nutricionais. Viçosa, MG: Universidade Federal de Viçosa, 2005. 186p.

SALVIANO, L.M.C.; NUNES, M.C.F.S. Feno de maniçoba na suplementação de novilhos alimentados com feno de capim buffel. Petrolina: EMBRAPA-CPATSA, 1991. 14p. (Boletim de Pesquisa, 38).

SIBBALD, I.R.; WOLYNETZ, M.S. Relationships between estimates of biovailable energy made with adult cockerels and chicks: effects of feed intake and nitrogen retention. Poultry Science, v.64, p.127-38, 1985.

SILVA, R.D.M.; NAKANO, M. Sistema caipira de criação de galinhas. Piracicaba: O Editor, 1998. 110p.

SILVA, D.J.; QUEIROZ, A.C. Análise de alimentos: métodos químicos e biológicos. 3.ed. Viçosa, MG: Editora UFV, 2002. $165 p$. 